\title{
Optimizing Dynamic Interactions between a Cardiac Patch and Inflammatory Host Cells
}

\author{
Donald O. Freytes $^{\mathrm{a}}$ Laura Santambrogio $^{\mathrm{b}}$ Gordana Vunjak-Novakovic ${ }^{\mathrm{a}}$ \\ Departments of ${ }^{a}$ Biomedical Engineering, Columbia University, and ${ }^{b}$ Pathology, Microbiology and Immunology, \\ Albert Einstein College of Medicine, New York, N.Y., USA
}

\section{Key Words}

Tissue engineering $\cdot$ Cardiogenesis $\cdot$ Cardiac patch $\cdot$ Stem cells $\cdot$ Inflammation $\cdot$ Bioreactor $\cdot$ Macrophages

\begin{abstract}
Damaged heart muscle has only a minimal ability for regeneration following myocardial infarction in which cardiomyocytes are lost to ischemia. The most clinically promising approach to regeneration of cardiac muscle currently under investigation is that of injecting cardiogenic repair cells or implanting a preformed tissue-engineered patch. While major advances are being made in the derivation of functional human cardiomyocytes and the development of tissue-en-
\end{abstract}

gineering modalities for cardiac repair, the host environment into which the repair cells are placed is largely overlooked. Within seconds of myocardial ischemia, hypoxia sets in in the myocardium and the inflammatory response starts, characterized by rapid deployment of circulating cells and the release of paracrine and autocrine signals. Therefore, the inflammatory conditions under which these interactions take place, the design of the scaffold material used, and the maturity of the implanted cells will determine the outcomes of any stem cell-based therapy. We discuss here the interactions between implanted and inflammatory cells of the host, which are critical for the design of effective heart repair therapies.

\section{Abbreviations used in this paper}

\begin{tabular}{|c|c|c|c|}
\hline ECM & extracellular matrix & MI & myocardial infarction \\
\hline ePTFE & expanded polytetrafluoroethylene & MIF & migration inhibitory factor \\
\hline FGF & fibroblast growth factor & MLC2A & myosin light chain $2 \mathrm{~A}$ \\
\hline hESCs & human embryonic stem cells & MMP & matrix metalloproteinase \\
\hline HIF & hypoxia-inducible factor & NGF & nerve growth factor \\
\hline hiPSCs & human-induced pluripotent stem cells & PMNCs & polymorphonuclear cells \\
\hline HLA & human leukocyte antigen & PVDF & polyvinylidene fluoride \\
\hline IGF & insulin-like growth factor & TGF & transforming growth factor \\
\hline IL & interleukin & TNF & tumor necrosis factor \\
\hline LIF & leukemia inhibitory factor & VEGF & vascular endothelial growth factor \\
\hline MCP & macrophage chemotactic protein & & \\
\hline
\end{tabular}

\section{KARGER}

() 2011 S. Karger AG, Basel

Fax +4161306 1234

E-Mail karger@karger.ch

www.karger.com
Accessible online at: www.karger.com/cto
Dr. Gordana Vunjak-Novakovic

Department of Biomedical Engineering, Columbia University

Vanderbilt Clinic, Room 12-234

622 West 168th Street, New York, NY 10032 (USA)

Tel. +1 212305 2304, E-Mail gv2131@ columbia.edu 
Fig. 1. Examples of two approaches to cardiac tissue engineering. Stem cells are differentiated into cardiac repair cells and seeded onto a scaffold material or mixed with an injectable matrix. The scaffold with the repair cells is placed at the site of MI or used to replace the infarcted tissue. Cells encapsulated within a hydrogel matrix are delivered to the site of injury via direct injection. Created using Servier Medical Art.

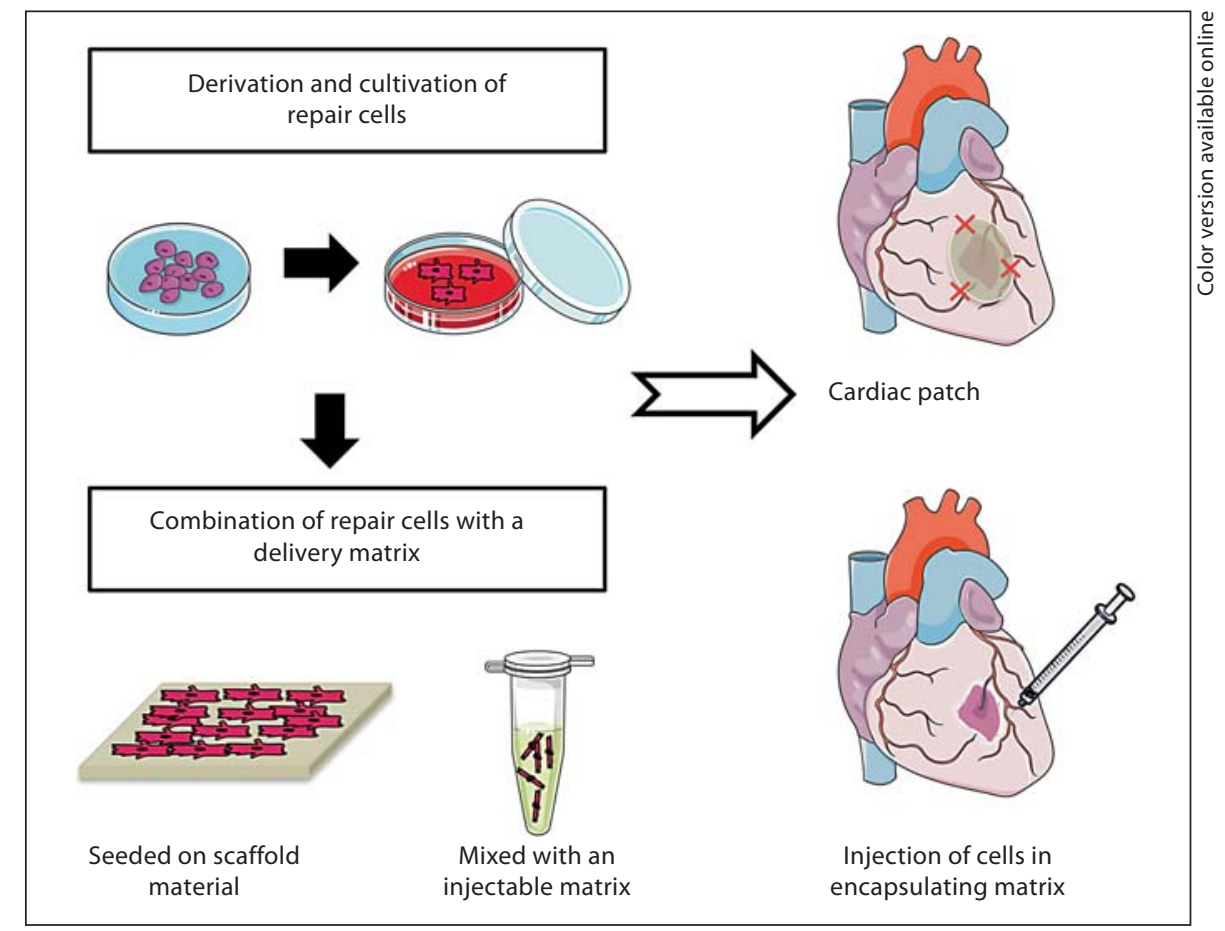

\section{Introduction}

Myocardial infarction (MI) remains one of the most prevalent forms of ischemic heart disease with a high long-term mortality rate due to heart failure. MI is currently the leading cause of death in the USA and industrialized nations, imposing an enormous health and economic burden on the patient and our aging population [Ostadal, 2009]. It is well established that the heart has only a minimal ability to regenerate itself following injury [Bergmann et al., 2009], making the development of effective therapies both necessary and challenging.

In most cases the ischemic region encompasses the entire thickness of the myocardium, forming a transmural infarct and leaving behind nonfunctional necrotic tissue that will eventually be replaced by a scar. The goal of cardiac therapy is to help regenerate damaged myocardium via cardiac repair cells injected into the damaged myocardium, implanted within an in vitro engineered cardiac patch, or recruited from the host. In all cases, it is necessary to provide a source of all three cardiac cell subpopulations - cardiac myocytes, endothelial cells, and cardiac fibroblasts - and to achieve cellular engraftment, electromechanical coupling, and ultimate incorporation into the host tissue and its vascular supply.
Approaches to cardiac tissue engineering range from the use of single cells to the construction of complex cellular constructs, with most effort focusing on: (i) injection of a cell suspension containing either purified or mixed populations of cells into the myocardium, and (ii) implantation of a cardiac patch that combines a scaffold component and a cellular component (fig. 1).

Cellular therapies based on intramuscular injections have had variable outcomes largely due to low cellular retention at the site of injection, poor survival of the injected cells, and poor cell incorporation with the host tissue [Laflamme and Murry, 2005]. The therapeutic effects achieved thus far by using cell suspensions have been related mostly to revascularization of infarcted myocardium and attributed to the effects of factors released by the cells rather than to cell engraftment. It is thought that cell retention at the site of delivery and cell function could be markedly improved by implanting an engineered patch that would eventually integrate and electrically couple with the native myocardium [Zimmermann et al., 2006; Vunjak-Novakovic et al., 2010b].

For both approaches, there is a need for a suitable source of repair cells. Several potential types of repair cells are being pursued, including mesenchymal stem cells, circulating progenitor cells, and resident cardiac progenitor cells. In this article, we focus on the use of em- 
Fig. 2. Dynamic and reciprocal interactions between the infarcted myocardium and the engineered cardiac patch. (1) Infiltrating cells will interact with the delivered cells via direct contact and/or secreted molecules. (2) Degradation products of the scaffold or matrix containing the cells will interact with the host tissue cells and infiltrating cells. (3) The repair cells will also interact with the healthy and necrotic myocardial tissue at the infarct site. Created using Servier Medical Art.

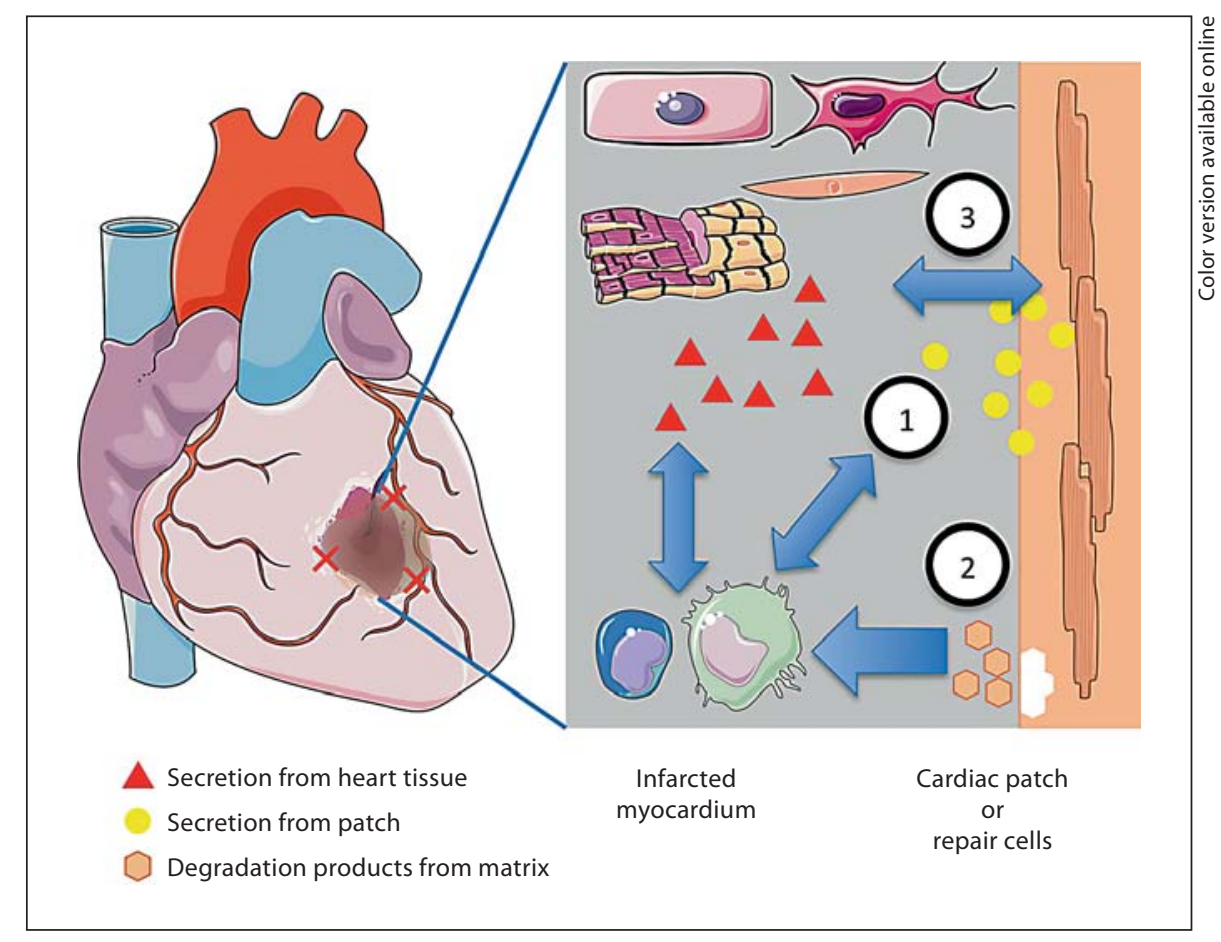

bryonic and pluripotent cells, which are the only cell types to date with the documented ability to give rise to bona fide cardiomyocytes.

The implanted repair cells, in the form of either a suspension or a contractile cardiac patch, are inevitably subjected to the inflammatory response at the site of infarction and the immune response elicited by the delivered cells. These interactions include a cross talk between infiltrating cells, healthy cardiac tissue, necrotic cardiac cells, and the cells within the engineered cardiac tissue (fig. 2). Although the dynamic interactions between the implanted cells and the host cells are likely to substantially affect the therapeutic outcomes, surprisingly little work has been done towards understanding the factors of interest and optimizing the conditions and timing of cell delivery. One reason for the lack of research in this area may be the scarcity of controllable in vitro models that can take into account the multiple factors of interest.

In this forward-looking review, we focus on the interactions between an implanted cardiac patch and the inflammatory cells of the host. Our laboratory, as well as several others, has found that the inflammatory environment present at the site of injury can both positively and negatively affect the outcomes of tissue-engineered heart repair [Laflamme et al., 2007; van Laake et al., 2008; Fer- nandes et al., 2010]. Notably, cell implantation in the setting of acute cardiac infarction gives better therapeutic outcomes compared to chronic cardiac infarction, suggesting that the inflammatory response and the presence of macrophages do not necessarily limit the repair processes. These experimental findings suggest an intriguing possibility that the inflammatory factors can in fact be harnessed to enhance repair.

We discuss the cellular events following MI and how these events can be mimicked in vitro towards a better understanding of the progression and effects of the inflammatory response on the repair cells. We propose that by developing robust and controllable in vitro testing platforms working with human cells, researchers will be able to systematically examine the survival of engineered patches over the course of the healing process before performing costly and difficult to control animal studies while leading the way to eventual translation to humans. Bioreactor platforms can be developed based on the current body of knowledge from in vitro studies and animal models. These platforms can help determine the optimum properties of the cardiac patch and an optimum time for implantation for maximal survival and efficacy of the repair cells and harnessing of the inflammatory response. 


\section{Inflammatory Response to MI}

Myocardial tissue repair following infarction is a complex and tightly regulated biological process. During cardiac ischemia, the heart undergoes a three-step healing process characterized by inflammatory, proliferative, and maturation phases. Understanding the events, the important cellular players, and the biophysical environment within the healing tissue is pivotal to the design of stem cell-derived cardiac patches aimed at achieving anatomical and functional tissue repair. One of the first events that take place following occlusion of a coronary artery is the development of an acute inflammatory response lasting approximately $7-10$ days and leading into the proliferative phase.

During the proliferative phase, cardiac fibroblasts are activated by the cytokine milieu - interleukin (IL)-1, IL8 , and tumor necrosis factor (TNF)- $\alpha$ - to synthesize new collagen and favor deposition of granulation tissue that consists mainly of a network of collagens I and III, and fibronectin [Dobaczewski et al., 2010]. During this phase, an increase in angiogenesis with neovascularization of the granulation tissue is observed.

The proliferative phase is followed by the remodeling phase where collagen is remodeled and realigned along tension lines and all the infiltrating inflammatory cells undergo apoptosis. Without intervention, this process can lead to remodeling of the myocardial wall and ultimately affect cardiac output. It is therefore important to design therapies that can revert the effects of scar tissue formation and restore function while surviving the acute inflammatory process.

\section{Early Cellular Events following Cardiac Ischemia}

Within seconds hypoxia sets in in the myocardium, and within minutes adenosine triphosphate (ATP) depletion of the cardiomyocytes leads to the cardiomyocyte's inability to contract. These initial events lead to the initial onset of necrosis and the beginning of the inflammatory response. During the inflammatory phase, dying cardiovascular cells release proinflammatory signals, leading to rapid infiltration of polymorphonuclear cells (PMNCs) that begin to clear cellular debris within the infarct and to secrete signaling molecules.

Within $1 \mathrm{~h}$ following cardiac infarction, PMNCs are recruited to the site of injury via secreted molecules from damaged endothelial cells and platelets and secrete a series of proteases that help degrade and turn over damaged extracellular matrix (ECM) proteins and facilitate phagocytosis of necrotic tissue debris. As such, PMNCs are the most prominent cells within the first couple of days following injury, and their number decreases shortly thereafter due to apoptosis [Jordan et al., 1999]. Figure 3 shows the appearance and progression of PMNCs at the site of infarction starting with the infiltration of neutrophils and monocytes from peripheral blood.

Infiltrating monocytes eventually differentiate into macrophages at the site of injury, where they phagocytose necrotic cells and enzymatically degrade the ECM. Macrophages and monocytes replace neutrophils as the most prominent cells around $48 \mathrm{~h}$ following MI [Lambert et al., 2008]. These macrophages phagocytose apoptosed neutrophils, leading to the release of cytokines such as transforming growth factor (TGF)- $\beta$, that initiate fibrotic tissue remodeling during the proliferative phase [Frangogiannis, 2008; Lambert et al., 2008]. Additionally, macrophages release cascades of cytokines and chemokines such as IL-1, IL- 6 , and TNF- $\alpha$ that are fundamental in resolving the tissue injury, stimulating granulation tissue and initiating the repair mechanisms [Lambert et al., 2008].

The infiltration of monocytes and their subsequent differentiation into macrophages can be divided into two progressive phases. In mice, the first phase recruits lymphocyte antigen 6 expressing monocytes (Ly-6Chi) via $\mathrm{C}-\mathrm{C}$ motif receptor 2 (CCR2) chemokines, while the second phase recruits $\mathrm{Ly}-6 \mathrm{C}^{\text {lo }}$ monocytes via $\mathrm{CX}_{3} \mathrm{C}$ motif receptor $1\left(\mathrm{CX}_{3} \mathrm{CR} 1\right)$ chemokines. In humans, a similar system has been observed with $\mathrm{Ly}-6 \mathrm{C}^{\text {hi }}$ and Ly- $6 \mathrm{C}^{\text {lo }}$ monocytes corresponding to $\mathrm{CD} 14^{+} \mathrm{CD} 16^{-}$and $\mathrm{CD}_{14}{ }^{+} \mathrm{CD} 16^{+}$, respectively [Tsujioka et al., 2009]. $\mathrm{CD}^{+} 4^{+} \mathrm{CD} 16^{-}$(Ly-6Chi) monocytes exhibit phagocytic, proteolytic, and inflammatory properties, while $\mathrm{CD}_{14}{ }^{+} \mathrm{CD} 16^{+}\left(\mathrm{Ly}-6 \mathrm{C}^{\mathrm{lo}}\right)$ monocytes promote healing via myofibroblast accumulation, angiogenesis, and matrix deposition [Nahrendorf et al., 2007].

Initially, classical activation of monocytes and macrophages takes place, leading to the production of nitric oxide (NO) and proinflammatory cytokines such as IL-1 $\beta$, IL-6, and TNF- $\alpha$. As the acute inflammatory response progresses there is a shift towards a more anti-inflammatory response over time. The dynamics and magnitude of changes in the presence of cytokines and cell populations during the inflammatory response imply that it is important to consider the type and maturity level of the cells implanted, the kind of matrix, and the length of the gap between implantation and the initiation of the inflammatory response. 
Fig. 3. Presence of inflammatory cells at the infarct site. Following MI there is a transient presence of different inflammatory cells such as neutrophils, monocytes, and polarized macrophages. Proinflammatory macrophages (M1) appear early during the inflammatory response and their number decreases over time with the eventual increase in anti-inflammatory macrophages (M2). Created using Servier Medical Art.

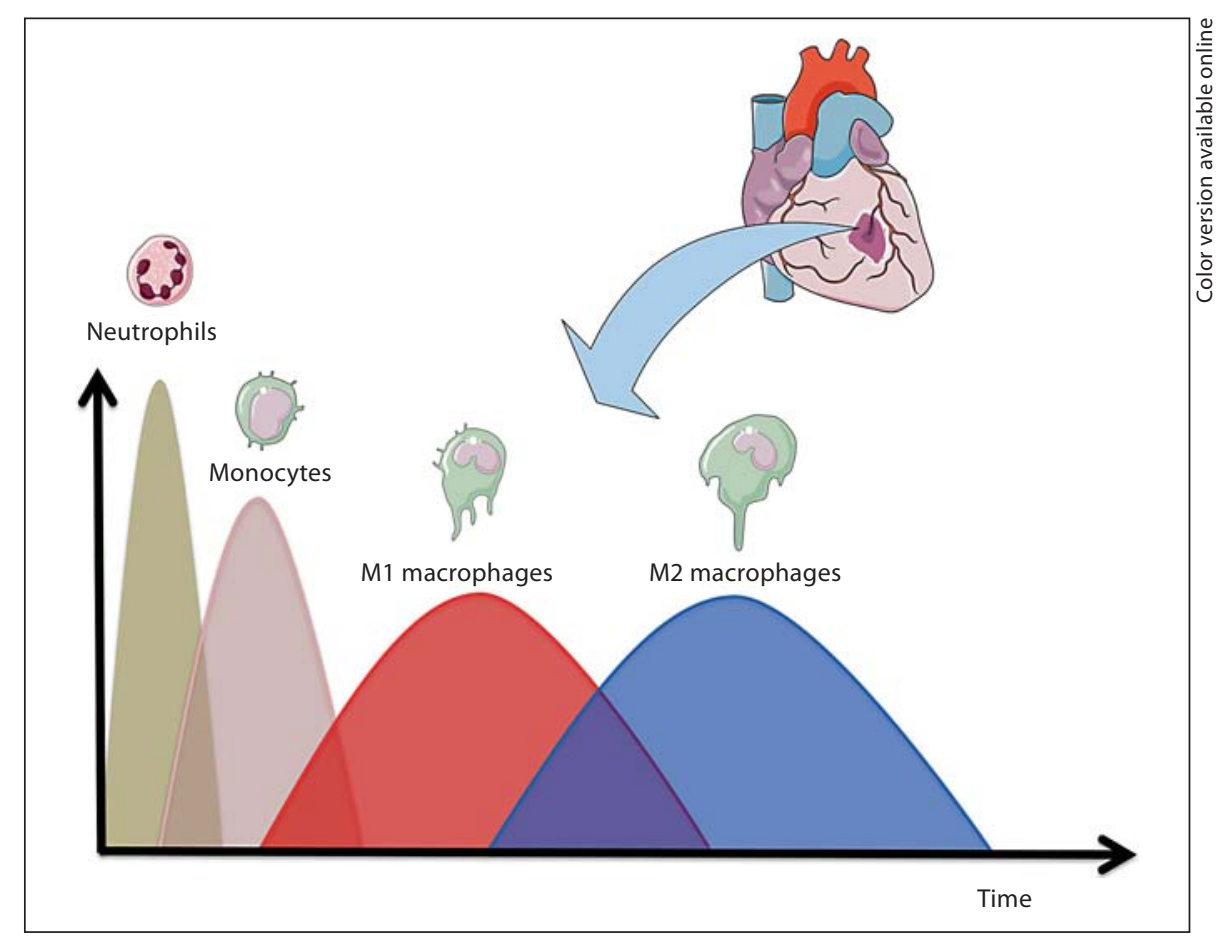

\section{Polarized Macrophages (M1 vs. M2)}

As shown in figure 3, macrophages can be polarized into proinflammatory (M1) macrophages and anti-inflammatory (M2) macrophages [Martinez et al., 2006; Nahrendorf et al., 2007; Lambert et al., 2008; Troidl et al., 2009]. M1 activation is typically associated with inflammation, tumor resistance, and graft rejection and followed by phase II with the recruitment of anti-inflammatory monocytes and their activation into antiinflammatory macrophages (alternative macrophage activation, M2). M2 macrophages exhibit a noninflammatory profile with the expression of arginase 1 and 2 and TGF- $\beta$, and their activation is associated with immunoregulation, matrix deposition, remodeling, and graft acceptance [Martinez et al., 2006; Nahrendorf et al., 2007; Brunelli and Rovere-Querini, 2008; Lambert et al., 2008].

Stated differently, M1 and M2 macrophages follow a bimodal response (fig. 3) in which early events are dominated by M1 macrophages and gradually shift towards an M2-dominated response [Troidl et al., 2009]. M2 macrophages turn over the ECM during scar formation and promote angiogenesis by activation of both resident and infiltrating endothelial and fibroblast cells.
The dynamic nature of these events leads to several questions:

- Is the timing of implantation important for survival of the repair cells?

- Does the inflammatory environment affect the repair cells within the infarct bed?

- Can the inflammatory response be harnessed to maximize the effectiveness of implanting engineered cardiac tissues?

\section{Cardiac Tissue Engineering}

The current knowledge on the effects of the molecular and biophysical factors involved in tissue formation is being translated into various systems for cardiac tissue engineering in vitro and in vivo. The overall goal of these efforts is to stimulate in situ recruitment of circulating and/or resident progenitor cells or to create functional cardiac tissue that can be used to improve the healing process or to completely replace damaged myocardium. Different laboratories have approached cardiac tissue engineering in different ways via:

- cultivation of scaffold-free stackable cell sheets [Shimizu et al., 2002], aggregates [Stevens et al., 2009a, b], and organoids [Chien et al., 2008]; 
- repopulation of decellularized native tissue [Ott et al., 2008];

- mechanical stimulation of cells in hydrogels [Zimmermann et al., 2002; Eschenhagen et al., 2006; Zimmermann et al., 2006];

- cell cultivation on perfused channeled scaffolds [Radisic et al., 2005, 2006];

- electrical stimulation of cells in porous scaffolds [Radisic et al., 2004; Chiu et al., 2008; Tandon et al., 2009], and

- cell delivery in injectable hydrogels [Atluri and Woo, 2008; Martens et al., 2009; Wollert and Drexler, 2010]. Regardless of the approach used, the current limitation - apart from any immune-rejection issue - is the availability of an abundant cell source of the necessary cell types.

\section{Repair Cells}

Although resident progenitor cells within the myocardium can contribute to healing, the cellular events that follow an MI may decrease the potential contributions of these cells in sufficient numbers to achieve complete myocardial healing. A cardiac patch should ideally provide mature and functional cardiomyocytes as well as supportive cardiac fibroblasts and endothelial cells that function mechanically and electrically in synchrony with the native myocardium. If no cardiomyocytes are used, an appropriate cell population could be used to promote resident stem cells to replace tissue or stimulate angiogenesis at the site of implantation. Cardiac progenitors cells could be harvested and expanded in vitro and represent an alternative cell population for cardiac tissue constructs [Vunjak-Novakovic et al., 2010a]. Even after finding an abundant cell source of cardiomyocytes and/ or repair cells, we will continue to face the challenge of maintaining the cells alive and functional after implantation by surviving the host tissue response.

Human embryonic stem cells (hESCs) and human induced pluripotent stem cells (hiPSCs) can give rise to all three cardiac cell populations [Dambrot et al., 2011; Kattman et al., 2011]. These cells can be expanded to sufficient cell numbers and, in principle, can differentiate into all cell types in the human body. Recent studies have resulted in multiple protocols that can produce functional cardiomyocytes from the existing hESC lines.

In one of these studies [Yang et al., 2008], a process was developed to systematically stage, in vitro, the events that occur in a developing embryo that give rise to cardiac cells. By exposing hESCs to cytokines and low oxygen, the three distinct mesoderm-derived populations were generated. Molecular induction of cardiovascular lineages in hESCs resulted in visible synchronous contractions, with $>50 \%$ of cells expressing cardiac troponin $\mathrm{T}$ and myosin light chain 2A (MLC2A), along with developmental cardiac markers (Nkx2.5, Isl1, Tbx5, Tbx20). Patch-clamp and microelectrode studies confirmed functionality through a dominant voltage-dependent potassium current ( $80 \%$ cells), electrical coupling, and beating profiles characteristic of cardiomyocytes [Yang et al., 2008]. The same overall approach has been recently extended to the derivation of cardiomyocytes from human iPS cells [Kattman et al., 2011] and their use in controlled studies of cardiac disease [Itzhaki et al., 2011; Masuda and Hanazono, 2011].

hESC-derived cardiomyocytes can be injected as a cellular suspension, encapsulated within a hydrogel matrix, or delivered within a cardiac patch (fig. 1). The delivery of hESC-derived cells to the heart tissue needs to provide means for the cells to survive the surgical placement, low oxygen and low nutrient conditions, and the host tissue response so that the cells can subsequently divide, migrate, and repopulate the damaged tissue. These are ambitious objectives that are hard to achieve even under tightly controlled laboratory conditions. Therefore, it is important to understand how various types of cardiac repair cells function and behave under normal physiological conditions and during cardiac ischemia and how they respond to the inflammatory cells of the host.

\section{Scaffold Material}

The scaffold material used to create a cardiac construct (synthetic or naturally occurring) can markedly affect the survival of the repair cells and the type of inflammatory response mounted by the host tissue. Synthetic materials commonly used for cardiac tissue engineering include polymers that can be used as an injectable hydrogel or manufactured into sheets (e.g. by electrospinning) providing mechanical support to the cultured cells [Sui et al., 2011]. The ultrastructure, composition, particle size, and topology of synthetic scaffold materials can also have adverse effects on cellular recruitment, infiltration, and complement activation, thus affecting the inflammatory response and the type of macrophage activation [Kou and Babensee, 2011; Sui et al., 2011]. For example, Paul et al. [2008] found that microtextured polyvinylidene fluoride (PVDF) surfaces signif- 
icantly affected macrophage activation when compared to nanotextured PVDF surfaces. In addition, Bota et al. [2010] showed that expanded polytetrafluoroethylene (ePTFE) materials elicited different proinflammatory responses as a function of pore size [Bota et al., 2010]. The use of collagen modified poly(2-hydroxyethyl methacrylate-co-methacrylic acid) hydrogels with an appropriate pore size $(30-40 \mu \mathrm{m})$ was shown to increase angiogenesis and elicit a shift towards M2 macrophages when implanted onto the myocardium [Madden et al., 2010]. These studies highlight the potential to modulate the host tissue response by engineering the scaffold material.

Naturally occurring biomaterials provide an alternative to synthetic scaffold materials. The ECM obtained by decellularization of tissues may provide an optimum scaffold material in terms of the composition and spatial arrangement of key components that help support cellular attachment, proliferation, and differentiation [Badylak et al., 2009]. Recent studies have shown that the process used to create the ECM scaffold material will affect the composition and the host tissue response elicited once implanted. Heavily cross-linked ECM scaffolds elicit a more rigorous inflammatory response associated with M1 macrophages such as the presence of CD80+ and CCR7+ cells when compared to non-cross-linked ECM scaffolds. In addition, non-cross-linked ECM showed a predominantly $\mathrm{M} 2$ response with constructive tissue remodeling after 16 weeks in a rat model [Valentin et al., 2009b].

Overall, the choice and design of the scaffold material used for a cardiac patch is important for the survival of any repair cell used but it could also potentially affect the environment the cells will be subjected to due to the type of inflammatory response elicited by the scaffold material itself. Since the inflammatory response is necessary for proper myocardial healing, further research is needed to determine the right balance required to maintain the physiological progression of the inflammatory response while tipping the balance towards achieving cardiac construct survival and integration.

\section{Implantation of Cardiac Grafts into the Infarct Bed}

Overall, cardiac tissue engineering aims to develop an engineered patch providing cardiomyocytes along with their supporting cells to improve the functionality of the postinfarct myocardium. At this time, several factors of cardiac patch implantations need to be optimized, including in particular: (i) the time frame for inserting the

Cardiac Patch and Inflammatory Host Cells cardiac patch, (ii) the cellular composition, and (iii) the level of maturity of the stem cells.

Regarding the time frame, it is likely that the insertion of a cardiac patch in the early inflammatory phase is not advisable. The presence of necrotic tissue could severely compromise the viability of the stem cells and limit cellular engraftment. Additionally, the incoming granulocytes secrete proteases to digest the ECM that could also decrease the survival of the patch and affect its mechanical integrity. Finally, the infiltrating macrophages release a series of proinflammatory cytokines that could potentially favor stem cell apoptosis and can lead to poor cellular engraftment.

On the other hand, it appears that placing a cardiac patch during the formation of granulation tissue could be relevant to increasing the number of newly formed cardiomyocytes and to decreasing scarring. In fact, the time between the formation of granulation tissue and remodeling is when the tissue is most plastic and could be remodeled to favor the formation of anatomically and physiologically functional cardiac tissue. hESC-derived cardiomyocytes have been shown to form stable myocardial grafts when injected into the healthy heart of athymic rats but not in infarcted myocardium [Laflamme et al., 2007]. This finding suggests that the inflammatory response could have a significant impact on graft acceptance and underscores the importance of studying the effects of the host tissue environment on the function, survival, and engraftment of the repair cells and patches.

In addition to the host tissue response, another obstacle to the development of effective heart therapies is the survival of the patch once implanted in the absence of a functioning vascular system. The cardiac patch needs to be engineered so that it survives in suboptimal conditions that differ in significant ways from the optimal conditions found in the laboratory. This means that the patch must survive the biophysical conditions (e.g. mechanical loading, electrical stimulation, oxygen concentrations) as well as the host tissue response (e.g. innate and immune response) present at the site of implantation.

Because the existing hESC lines are derived from a handful of individuals with unique histocompatibility complexes, the host tissue would most likely reject any cardiac patch derived from any of the existing hESC cell lines unless tolerance is induced prior to implantation [Fairchild et al., 2007; Robertson et al., 2007]. Generation of an autologous cardiac patch, using iPS cells, requires that the appropriate cells be previously prepared from the patient's cells, stored, expanded, and differentiated into cardiac lineages. The major advantage of using autolo- 
gous stem cells is obviously the absence of tissue rejection since there are no major histocompatibility differences between the donor and the recipient. However, hESC-derived cells can still be used to study the interactions between the host tissue response and the cardiac repair cells. The limitations of using an allogeneic system over an autologous system come into play when direct cell contact between cells is required.

Generation of heterologous cardiac patches requires storage of previously collected and expanded stem cells from healthy donors with a known human leukocyte antigen (HLA) type. The procedure would basically reproduce what is currently done for storage and HLA (HLA-A, B, and $C$ and HLA-DR, DP and DQ) typing of marrow cells used in bone marrow transplants. The obvious advantage would be that a sufficient amount of previously stored cells could be thawed and used for the preparation of the cardiac patch in a 'just in time' manner. The biggest disadvantage is that only a close but never perfect HLA match (unless the patient has an identical twin) can be achieved. In this case the recipient would require long-term immunosuppressive therapy, a risk that patients normally take following bone marrow transplantation to treat cancer.

\section{In vitro Models of the Interactions between a Cardiac Patch and the Inflammatory Environment}

Although the inflammatory response is a complex and dynamic system, there are key events that can be mimicked in vitro such as the transient appearance of distinct cell types and the biophysical stimuli experienced by the cells. A cardiac patch can be cultured in the presence of neutrophils, monocytes, and macrophages at different time intervals recreating to some extent the cellular population present at the time of implantation and the cellular population most likely to appear as the inflammatory response progress.

There are multiple sources of peripheral blood mononuclear cells including donor blood [Repnik et al., 2003] samples and established cell lines [Auwerx, 1991]. Common cell lines such as HL-60 and THP-1 that produce neutrophils and monocytes like cells in culture can be used. However, these are cells derived from leukemic cells and can behave differently compared to freshly isolated leukocytes [Auwerx, 1991]. The exposure of the patch to inflammatory cells can be via direct contact in nature, allowing for cell-cell interactions to occur in addition to paracrine and autocrine signaling. Alternatively, paracrine and autocrine signaling can be studied using trans- well systems or using a permeable membrane to physically separate each cell type. Commercially available trans-wells can be used with standard well plates that allow for physical separation of the cells while permitting molecules to diffuse freely between the chambers and can be imaged using conventional microscopes.

Recently, there have been advances in the design of bioreactors which can impart complex biophysical stimuli on the cells and can be used to closely mimic the environment found in vivo [Vunjak-Novakovic and Scadden, 2011]. The novelty of these bioreactors is that they allow us to grow and train the cardiac patch in the laboratory by providing the physical stimuli (e.g. hydrodynamic, electrical, mechanical) that will be experienced after implantation. Conditioning of the cardiac cells and engineered patches could increase their survival after implantation and help us understand how biophysical stimuli affect the maturation and function of the potential repair cells. Other conditions can be added to better mimic the pathological conditions under which they will be required to function.

A bioreactor can be designed to include hypoxic conditions that normally follow coronary occlusion and a subsequent inflammatory response by permitting the transient exposure to different cell types normally found at the ischemic injury. Hypoxia is inherent to the infarcted myocardium, and it directly modulates the host tissue response at the site of implantation. A previous study showed that monocytes activated by transient hypoxia can have a cardioprotective effect during ischemia and reoxygenation conditions [DaCosta et al., 2003]. Macrophages migrate and accumulate at sites with low oxygen tension and respond to hypoxia by upregulation of hypoxia-inducible factor (HIF)-1 and HIF-2, vascular endothelial growth factor (VEGF), fibroblast growth factor (FGF)-2, monocyte chemoattractant protein (MCP)-1, matrix metalloproteases (MMPs), and TNF- $\alpha$ [Negus et al., 1998; Murdoch et al., 2005]. Importantly, cardiomyocytes are known to increase expression of macrophage migration inhibitory factor (MIF) when exposed to hypoxic conditions [Jian et al., 2009].

The inflammatory conditions, along with the electrical and mechanical stimuli normally present in the heart, can be provided by a bioreactor designed to mimic the host tissue response and the biophysical environment experienced by the cardiac patch. By employing the current knowledge acquired using small animal and large animal models, targeted and high-throughput experiments can be performed recreating some of these complex events in vitro and substantially increasing the amount of infor- 
Fig. 4. Screening of platforms for optimization of key parameters in cardiac tissue engineering. Screening platforms can use the knowledge gained from in vivo testing in small animals (such as the sequential presence of inflammatory cells) to build more accurate screening platforms that take into account not only the biophysical stimuli present but also the host tissue response. The information gained can be validated in using large animal models that more closely resemble the physiological size and mechanical loading of the human heart. The fundamental goal is to build accurate screening platforms using human cells to predict the potential survival and function of engineered cardiac tissues. Ultimately the objective is to harness the host tissue response and repair the damaged tissue. Created using Servier Medical Art.

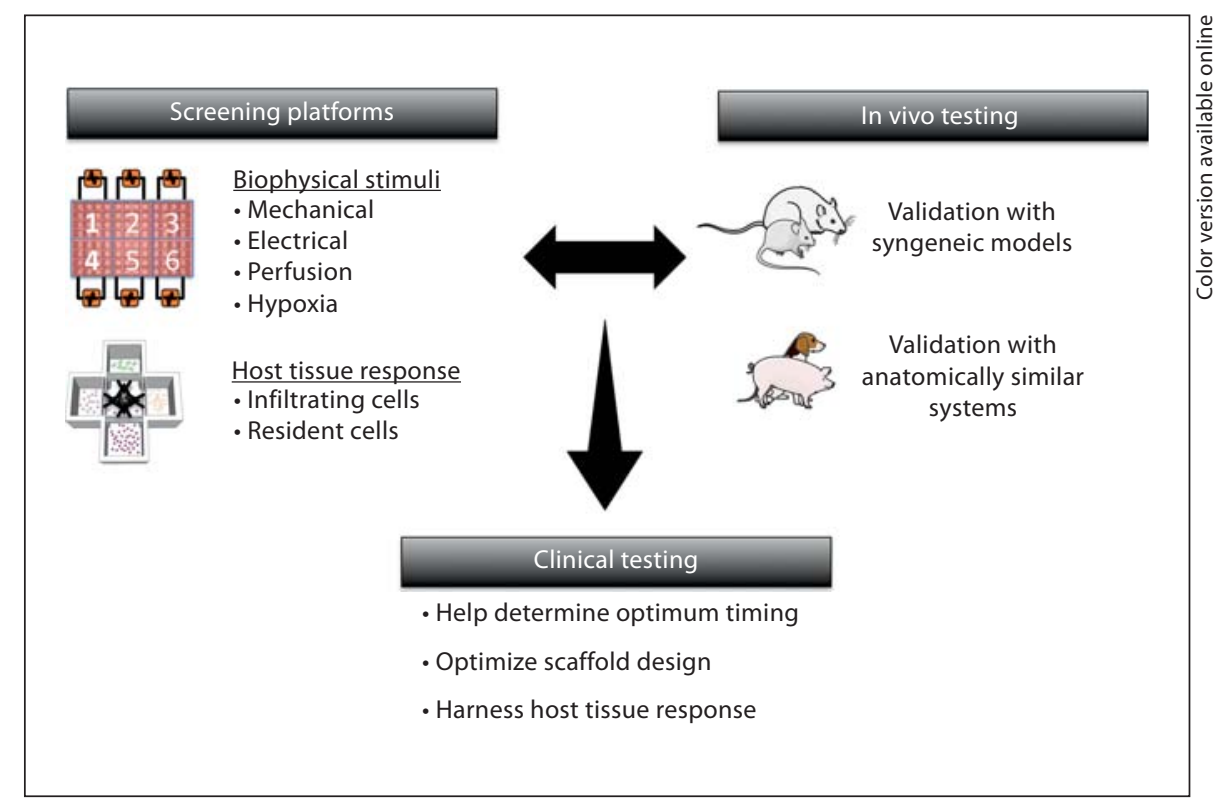

mation gained from in vitro models (fig. 4). Accurate in vitro models could lead to more efficient and less expensive animal testing and better translation into human therapies.

If direct contact between the leukocytes and the cardiac patch is not required, an allogeneic culture system will suffice and experiments can be performed using freshly isolated peripheral blood mononuclear cells. However, direct contact between leukocytes and the cardiac patch is often needed to truly recreate the inflammatory response in vitro. In such a case, the availability of an autologous system becomes a limiting factor. Even in the absence of $\mathrm{T}$ cells, macrophages can be activated by nonself cells and induce rejection [Ascher et al., 1984]. To overcome this problem one could perform experiments using cells from syngeneic animals. In a human model system, there are limited options to address this problem. Theoretically, hiPSCs and multiple blood donations could be obtained from a single donor. Even though this is possible in principle, the logistics and regulatory hurdles make this approach costly and complicated. Another approach would be to derive the appropriate leukocytes and cardiomyocytes from the same embryonic stem cell line. Although this is theoretically possible, there are no established protocols at this time to obtain leukocytes from hESCs cells.

Finally, studies of human cells can only be conducted in immunocompromised animals. The predictability of these studies is limited not only by a species mismatch (as in the case of using human cells in rodents) but also by the differences in size and physiology [Schwartz, 1998; Abarbanell et al., 2010]. In addition, there are documented differences in the ability of cytokines to activate receptors of other species if less than $60 \%$ of the amino acid identity is not retained [Scheerlinck, 1999]. Finally, physiological discrepancies between the heart rate of different species $(\sim 300-500$ beats/min for rodents vs. $\sim 60-80$ beats/min for humans) [Ytrehus, 2000] require further testing since they can potentially skew the effects observed when using human cells in rodent models.

\section{Harnessing the Inflammatory Response}

There is a growing body of evidence that the presence of monocytes and macrophages is important for the healing of myocardial tissue [Lambert et al., 2008]. Preventing the recruitment of monocytes via physical blockage and chemical depletion, or in genetically modified animal models, leads to poor healing and - in cases of $\mathrm{MI}$ - to increased ventricular remodeling [van Amerongen et al., 2007; Frangogiannis, 2008; Lambert et al., 2008; Wernli et al., 2009]. Macrophage inhibition via administration of chlodronate leads to a decrease in sympathetic hyperinnervation postinfarct with decreased levels of nerve growth factor (NGF) [Wernli et al., 2009]. Even secretion from proinflammatory macrophages at the infarct bed could affect the repair cells and the surrounding 
Fig. 5. Harnessing of the inflammatory response to improve the survival and engraftment of engineered cardiac tissues. (1) Chemotactic molecules that recruit supportive inflammatory cells can be encapsulated within the scaffold material and controlled-released into the wound site. (2) Alone or in combination with chemotactic molecules, inhibitory molecules can be encapsulated within the scaffold material to inhibit any cell that does not support the survival and engraftment of the patch. (3) The choice of scaffold material, along with its porosity and composition, and the degradation products that result from the host tissue response mediated breakdown could also provide stimulatory or inhibitory signals. Individually or in combination, the mechanisms shown can be used to harness the inflammatory environment leading to an optimized engineered cardiac patch. Created using Servier Medical Art.

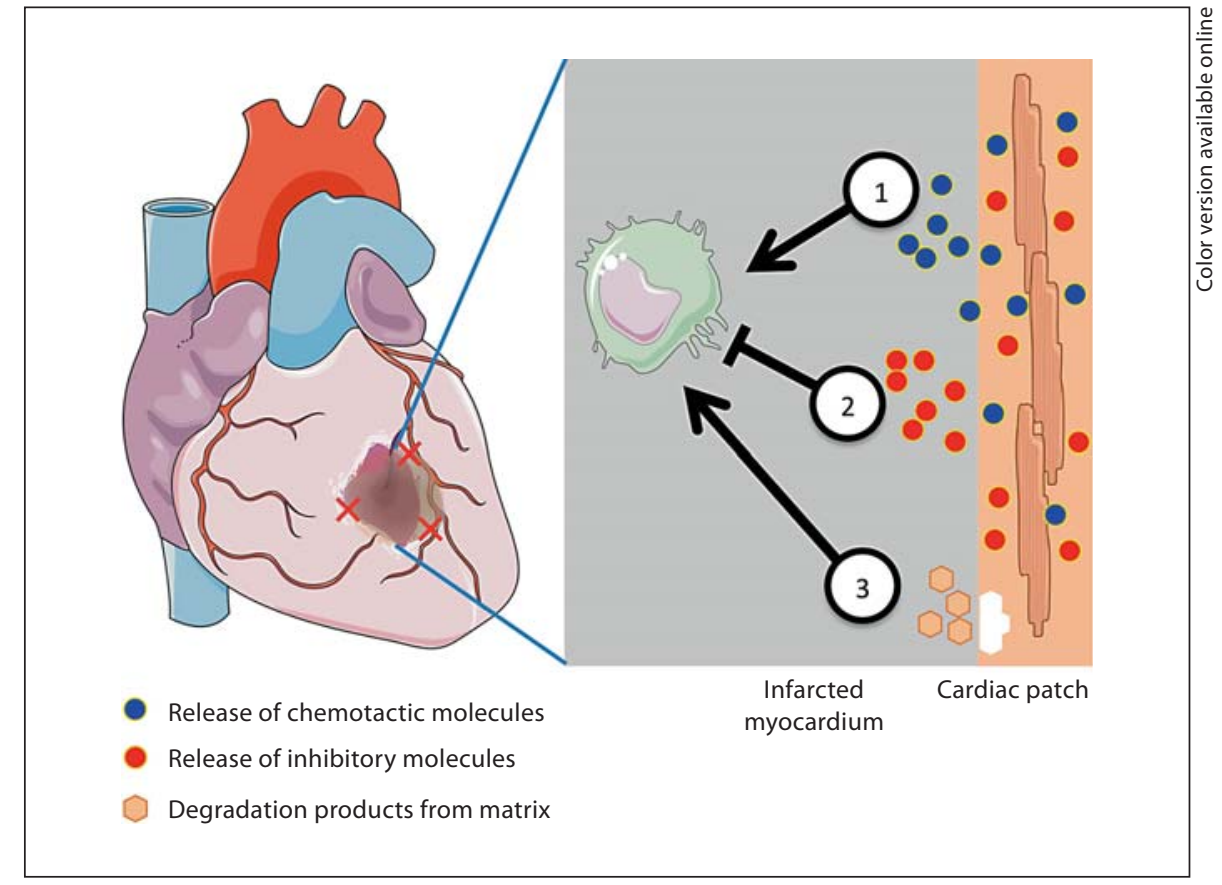

myocytes via an NO-dependent reduction of cardiac function [Joe et al., 1998]. Clearly these are important cells for resolution of the inflammatory response and are key players in the final outcome following an infarct.

In addition, degradation products of the scaffold components used to create the cardiac patch can also initiate recruitment and activation of macrophages at the wound site [Trial et al., 2004]. Fibronectin fragments have been shown to stimulate macrophages to release factors that protect hypoxic myocytes from apoptosis, leading to increased macrophage production of TNF- $\alpha$, FGF-1, insulin-like growth factor (IGF-1), and leukemia inhibitory factor (LIF) [Trial et al., 2004]. Chemically treated ECM scaffolds have been shown to recruit proinflammatory macrophages, while untreated scaffolds recruited antiinflammatory macrophages to the site of injury. Consistently, wounds treated with non-cross-linked ECM scaffolds showed more constructive remodeling when compared to cross-linked ECM scaffold-treated wounds [Badylak and Gilbert, 2008; Badylak et al., 2008; Valentin et al., 2009a]. Even a scaffold's topology can affect macrophage polarization [Bota et al., 2010], accentuating the importance of the scaffold choice and its potential effect on tissue repair.

These studies underscore the important roles of inflammatory cells, and in particular macrophages, in the healing response of ischemic myocardium. The effects observed thus far strongly suggest the possibility of harnessing the inflammatory response in order to maximize the survival of the delivered cells. Once the optimum inflammatory environment has been established, the timing of delivery and the delivery mechanism can be adjusted. In the latter case, controlled release of a chemotactic or inhibitory molecule targeted towards a specific cell population can be used as shown in figure 5. Also, the choice of scaffold material that promotes the desired inflammatory environment can be combined with other factors such as timing to create an optimal cardiac patch therapy.

\section{Concluding Remarks}

In summary, advances in stem cell biology and tissue engineering are critically important for the development of effective modalities for treating MI, which remains the leading cause of death in the developed world. At this time, the survival and function of cardiac grafts remain poor for at least two reasons: (i) tissue-engineering strategies do not provide the necessary cell protection and conditioning, and (ii) the ischemic/inflammatory environment of the injured heart is largely overlooked when determining the properties of cardiac grafts. 
Dynamic interactions between the implanted cardiogenic cells and the inflammatory milieu of MI can be investigated in vitro using a model of a cardiac patch in an inflammatory environment. For such a model to be a practical tool, robust and controllable in vitro testing platforms are designed to study cell populations of different compositions and maturity, cardiac patch properties, the use of cardioprotective factors, and the presence of specific inflammatory cells. In particular, in vitro platforms can be used to design a cardiac patch with the best capability for survival and function following implantation into the infarct bed and to determine the timing of implantation for best utilization of inflammatory factors.
We proposed that some of the existing barriers for the development of effective cell therapies for MI could be overcome by manipulating the properties of the implanted patch cardiac cells and their interactions with the host environment. One particularly intriguing possibility is that the inflammatory factors in the host environment could be harnessed to accelerate and improve the tissueengineered regeneration of the infarcted heart.

\section{Acknowledgements}

This work was funded by NIH grants (HL076485, EB002520, RR026244), New York State (C026721, C026449), and the Helmsley Foundation.

\section{References}

Abarbanell, A.M., J.L. Herrmann, B.R. Weil, Y. Chien, K.R., I.J. Domian, K.K. Parker (2008) Wang, J. Tan, S.P. Moberly, J.W. Fiege, D.R. Meldrum (2010) Animal models of myocardial and vascular injury. J Surg Res 162: 239249.

Ascher, N.L., R.A. Hoffman, D.W. Hanto, R.L. Simmons (1984) Cellular basis of allograft rejection. Immunol Rev 77: 217-232.

Atluri, P., Y.J. Woo (2008) Pro-angiogenic cytokines as cardiovascular therapeutics: assessing the potential. BioDrugs 22: 209-222.

-Auwerx, J. (1991) The human leukemia cell line, THP-1: a multifacetted model for the study of monocyte-macrophage differentiation. Experientia 47: 22-31.

Badylak, S.F., D.O. Freytes, T.W. Gilbert (2009) Extracellular matrix as a biological scaffold material: structure and function. Acta Biomater 5: 1-13.

Badylak, S.F., T.W. Gilbert (2008) Immune response to biologic scaffold materials. Semin Immunol 20: 109-116.

Badylak, S.F., J.E. Valentin, A.K. Ravindra, G.P. McCabe, A.M. Stewart-Akers (2008) Macrophage phenotype as a determinant of biologic scaffold remodeling. Tissue Eng Part A 14: 1835-1842.

-Bergmann, O., R.D. Bhardwaj, S. Bernard, S. Zdunek, F. Barnabe-Heider, S. Walsh, J. Zupicich, K. Alkass, B.A. Buchholz, H. Druid, S. Jovinge, J. Frisen (2009) Evidence for cardiomyocyte renewal in humans. Science 324 : 98-102.

Bota, P.C., A.M. Collie, P. Puolakkainen, R.B. Vernon, E.H. Sage, B.D. Ratner, P.S. Stayton (2010) Biomaterial topography alters healing in vivo and monocyte/macrophage activation in vitro. J Biomed Mater Res A 95: 649657.

Brunelli, S., P. Rovere-Querini (2008) The immune system and the repair of skeletal muscle. Pharmacol Res 58: 117-121. Cardiogenesis and the complex biology of regenerative cardiovascular medicine. Science 322: 1494-1497.

Chiu, L.L., R.K. Iyer, J.P. King, M. Radisic (2008) Biphasic electrical field stimulation AIDS in tissue engineering of multicell-type cardiac organoids. Tissue Eng Part A 17: 1465-1477.

DaCosta, M.L., Z. Yao, B.C. MacPherson, D.V. Jayakar, V. Jeevanandam (2003) Brief hypoxia conditions monocytes to protect reperfused cardiocytes against cell death via the CD11b receptor. J Heart Lung Transplant 22: 979-985.

Dambrot, C., R. Passier, D. Atsma, C.L. Mummery (2011) Cardiomyocyte differentiation of pluripotent stem cells and their use as cardiac disease models. Biochem J 434: 25-35.

Dobaczewski, M., C. Gonzalez-Quesada, N.G. Frangogiannis (2010) The extracellular matrix as a modulator of the inflammatory and reparative response following myocardial infarction. J Mol Cell Cardiol 48: 504-511.

Eschenhagen, T., W.H. Zimmermann, A.G. Kleber (2006) Electrical coupling of cardiac myocyte cell sheets to the heart. Circ Res 98: 573-575.

Fairchild, P.J., N.J. Robertson, S.L. Minger, H. Waldmann (2007) Embryonic stem cells: protecting pluripotency from alloreactivity. Curr Opin Immunol 19: 596-602.

Fernandes, S., A.V. Naumova, W.Z. Zhu, M.A. Laflamme, J. Gold, C.E. Murry (2010) Human embryonic stem cell-derived cardiomyocytes engraft but do not alter cardiac remodeling after chronic infarction in rats. J Mol Cell Cardiol 49: 941-949.

Frangogiannis, N.G. (2008) The immune system and cardiac repair. Pharmacol Res 58: 88111.
Itzhaki, I., L. Maizels, I. Huber, L. Zwi-Dantsis, O. Caspi, A. Winterstern, O. Feldman, A. Gepstein, G. Arbel, H. Hammerman, M. Boulos, L. Gepstein (2011) Modelling the long QT syndrome with induced pluripotent stem cells. Nature 471: 225-229.

-Jian, Z., J.B. Li, R.Y. Ma, L. Chen, Q.J. Zhong, X.F. Wang, W. Wang, Y. Hong, Y.B. Xiao (2009) Increase of macrophage migration inhibitory factor (MIF) expression in cardiomyocytes during chronic hypoxia. Clin Chim Acta 405: 132-138.

Joe, E.K., A.E. Schussheim, D. Longrois, T. Maki, R.A. Kelly, T.W. Smith, J.L. Balligand (1998) Regulation of cardiac myocyte contractile function by inducible nitric oxide synthase (iNOS): mechanisms of contractile depression by nitric oxide. J Mol Cell Cardiol 30: 303-315.

Jordan, J.E., Z.Q. Zhao, J. Vinten-Johansen (1999) The role of neutrophils in myocardial ischemia-reperfusion injury. Cardiovasc Res 43: 860-878.

Kattman, S.J., A.D. Witty, M. Gagliardi, N.C. Dubois, M. Niapour, A. Hotta, J. Ellis, G. Keller (2011) Stage-specific optimization of activin/nodal and BMP signaling promotes cardiac differentiation of mouse and human pluripotent stem cell lines. Cell Stem Cell 8: 228-240.

Kou, P.M., J.E. Babensee (2011) Macrophage and dendritic cell phenotypic diversity in the context of biomaterials. J Biomed Mater Res A 96: $239-260$.

Laflamme, M.A., K.Y. Chen, A.V. Naumova, V. Muskheli, J.A. Fugate, S.K. Dupras, H. Reinecke, C. Xu, M. Hassanipour, S. Police, C. O'Sullivan, L. Collins, Y. Chen, E. Minami, E.A. Gill, S. Ueno, C. Yuan, J. Gold, C.E. Murry (2007) Cardiomyocytes derived from human embryonic stem cells in pro-survival factors enhance function of infarcted rat hearts. Nat Biotechnol 25: 1015-1024. 
Laflamme, M.A., C.E. Murry (2005) Regenerating the heart. Nat Biotechnol 23: 845-856.

Lambert, J.M., E.F. Lopez, M.L. Lindsey (2008) Macrophage roles following myocardial infarction. Int J Cardiol 130: 147-158.

Madden, L.R., D.J. Mortisen, E.M. Sussman, S.K. Dupras, J.A. Fugate, J.L. Cuy, K.D. Hauch, M.A. Laflamme, C.E. Murry, B.D. Ratner (2010) Proangiogenic scaffolds as functional templates for cardiac tissue engineering. Proc Natl Acad Sci USA 107: 15211-15216.

Martens, T.P., A.F. Godier, J.J. Parks, L.Q. Wan, M.S. Koeckert, G.M. Eng, B.I. Hudson, W. Sherman, G. Vunjak-Novakovic (2009) Percutaneous cell delivery into the heart using hydrogels polymerizing in situ. Cell Transplant 18: 297-304.

Martinez, F.O., S. Gordon, M. Locati, A. Mantovani (2006) Transcriptional profiling of the human monocyte-to-macrophage differentiation and polarization: new molecules and patterns of gene expression. J Immunol 177: 7303-7311.

-Masuda, S., Y. Hanazono (2011) Induced pluripotent stem cells in long-QT syndrome. N Engl J Med 364: 181, author reply 181-182.

Murdoch, C., M. Muthana, C.E. Lewis (2005) Hypoxia regulates macrophage functions in inflammation. J Immunol 175: 6257-6263.

Nahrendorf, M., F.K. Swirski, E. Aikawa, L. Stangenberg, T. Wurdinger, J.L. Figueiredo, P. Libby, R. Weissleder, M.J. Pittet (2007) The healing myocardium sequentially mobilizes two monocyte subsets with divergent and complementary functions: J Exp Med 204: 3037-3047.

Negus, R.P., L. Turner, F. Burke, F.R. Balkwill (1998) Hypoxia down-regulates MCP-1 expression: implications for macrophage distribution in tumors. J Leukoc Biol 63: 758765.

Ostadal, B. (2009) The past, the present and the future of experimental research on myocardial ischemia and protection. Pharmacol Rep 61: 3-12.

Ott, H.C., T.S. Matthiesen, S.K. Goh, L.D. Black, S.M. Kren, T.I. Netoff, D.A. Taylor (2008) Perfusion-decellularized matrix: using nature's platform to engineer a bioartificial heart. Nat Med 14: 213-221.

Paul, N.E., C. Skazik, M. Harwardt, M. Bartneck, B. Denecke, D. Klee, J. Salber, G. ZwadloKlarwasser (2008) Topographical control of human macrophages by a regularly microstructured polyvinylidene fluoride surface. Biomaterials 29: 4056-4064.

Radisic, M., W. Deen, R. Langer, G. Vunjak-Novakovic (2005) Mathematical model of oxygen distribution in engineered cardiac tissue with parallel channel array perfused with culture medium containing oxygen carriers. Am J Physiol Heart Circ Physiol 288: H1278H1289.
Radisic, M., H. Park, F. Chen, J.E. Salazar-Lazzaro, Y. Wang, R. Dennis, R. Langer, L.E. Freed, G. Vunjak-Novakovic (2006) Biomimetic approach to cardiac tissue engineering: oxygen carriers and channeled scaffolds. Tissue Eng 12: 2077-2091.

Radisic, M., H. Park, H. Shing, T. Consi, F.J. Schoen, R. Langer, L.E. Freed, G. VunjakNovakovic (2004) Functional assembly of engineered myocardium by electrical stimulation of cardiac myocytes cultured on scaffolds. Proc Natl Acad Sci USA 101: 1812918134.

Repnik, U., M. Knezevic, M. Jeras (2003) Simple and cost-effective isolation of monocytes from buffy coats. J Immunol Methods 278: 283-292.

Robertson, N.J., F.A. Brook, R.L. Gardner, S.P. Cobbold, H. Waldmann, P.J. Fairchild (2007) Embryonic stem cell-derived tissues are immunogenic but their inherent immune privilege promotes the induction of tolerance. Proc Natl Acad Sci USA 104: 20920-20925.

-Scheerlinck, J.P. (1999) Functional and structural comparison of cytokines in different species. Vet Immunol Immunopathol 72: 39-44.

Schwartz, P.J. (1998) Do animal models have clinical value? Am J Cardiol 81: 14D-20D.

Shimizu, T., M. Yamato, Y. Isoi, T. Akutsu, T. Setomaru, K. Abe, A. Kikuchi, M. Umezu, T. Okano (2002) Fabrication of pulsatile cardiac tissue grafts using a novel 3-dimensional cell sheet manipulation technique and temperature-responsive cell culture surfaces. Circ Res 90: e40.

-Stevens, K.R., K.L. Kreutziger, S.K. Dupras, F.S. Korte, M. Regnier, V. Muskheli, M.B. Nourse, K. Bendixen, H. Reinecke, C.E. Murry (2009a) Physiological function and transplantation of scaffold-free and vascularized human cardiac muscle tissue. Proc Natl Acad Sci USA 106: 16568-16573.

Stevens, K.R., L. Pabon, V. Muskheli, C.E. Murry (2009b) Scaffold-free human cardiac tissue patch created from embryonic stem cells. Tissue Eng Part A 15: 1211-1222.

Sui, R., X. Liao, X. Zhou, Q. Tan (2011) The current status of engineering myocardial tissue. Stem Cell Rev 7: 172-180.

Tandon, N., C. Cannizzaro, P.H. Chao, R. Maidhof, A. Marsano, H.T. Au, M. Radisic, G. Vunjak-Novakovic (2009) Electrical stimulation systems for cardiac tissue engineering. Nat Protoc 4: 155-173.

Trial, J., R.D. Rossen, J. Rubio, A.A. Knowlton (2004) Inflammation and ischemia: macrophages activated by fibronectin fragments enhance the survival of injured cardiac myocytes. Exp Biol Med (Maywood) 229: 538545.

Troidl, C., H. Mollmann, H. Nef, F. Masseli, S. Voss, S. Szardien, M. Willmer, A. Rolf, J. Rixe, K. Troidl, S. Kostin, C. Hamm, A. Elsasser (2009) Classically and alternatively activated macrophages contribute to tissue remodelling after myocardial infarction. J Cell Mol Med 13: 3485-3496.
Tsujioka, H., T. Imanishi, H. Ikejima, A. Kuroi, S. Takarada, T. Tanimoto, H. Kitabata, K. Okochi, Y. Arita, K. Ishibashi, K. Komukai, H. Kataiwa, N. Nakamura, K. Hirata, A. Tanaka, T. Akasaka (2009) Impact of heterogeneity of human peripheral blood monocyte subsets on myocardial salvage in patients with primary acute myocardial infarction. J Am Coll Cardiol 54: 130-138.

Valentin, J.E., A.M. Stewart-Akers, T.W. Gilbert, S.F. Badylak (2009b) Macrophage participation in the degradation and remodeling of extracellular matrix scaffolds. Tissue Eng Part A 15: 1687-1694.

van Amerongen, M.J., M.C. Harmsen, N. van Rooijen, A.H. Petersen, M.J. van Luyn (2007) Macrophage depletion impairs wound healing and increases left ventricular remodeling after myocardial injury in mice. Am J Pathol 170: 818-829.

van Laake, L.W., R. Passier, P.A. Doevendans, C.L. Mummery (2008) Human embryonic stem cell-derived cardiomyocytes and cardiac repair in rodents. Circ Res 102: 10081010.

Vunjak-Novakovic, G., K.O. Lui, N. Tandon, K.R. Chien (2010a) Bioengineering heart muscle: a paradigm for regenerative medicine. Annu Rev Biomed Eng 13: 245-267.

Vunjak-Novakovic, G., D.T. Scadden (2011) Biomimetic platforms for human stem cell research. Cell Stem Cell 8: 252-261.

Vunjak-Novakovic, G., N. Tandon, A. Godier, R. Maidhof, A. Marsano, T.P. Martens, M. Radisic (2010b) Challenges in cardiac tissue engineering. Tissue Eng Part B Rev 16: 169-187.

Wernli, G., W. Hasan, A. Bhattacherjee, N. van Rooijen, P.G. Smith (2009) Macrophage depletion suppresses sympathetic hyperinnervation following myocardial infarction. Basic Res Cardiol 104: 681-693.

-Wollert, K.C., H. Drexler (2010) Cell therapy for the treatment of coronary heart disease: a critical appraisal. Nat Rev Cardiol 7: 204215.

-Yang, L., M.H. Soonpaa, E.D. Adler, T.K. Roepke, S.J. Kattman, M. Kennedy, E. Henckaerts, K. Bonham, G.W. Abbott, R.M. Linden, L.J. Field, G.M. Keller (2008) Human cardiovascular progenitor cells develop from a KDR+ embryonic-stem-cell-derived population. Nature 453: 524-528.

-Ytrehus, K. (2000) The ischemic heart - experimental models. Pharmacol Res 42: 193-203.

Zimmermann, W.H., I. Melnychenko, G. Wasmeier, M. Didie, H. Naito, U. Nixdorff, A. Hess, L. Budinsky, K. Brune, B. Michaelis, S. Dhein, A. Schwoerer, H. Ehmke, T. Eschenhagen (2006) Engineered heart tissue grafts improve systolic and diastolic function in infarcted rat hearts. Nat Med 12: 452-458.

Zimmermann, W.H., K. Schneiderbanger, P. Schubert, M. Didie, F. Munzel, J.F. Heubach, S. Kostin, W.L. Neuhuber, T. Eschenhagen (2002) Tissue engineering of a differentiated cardiac muscle construct. Circ Res 90: 223230. 